Pacific Journal of Mathematic 


\title{
INVARIANT MEASURES AND CESÀRO SUMMABILITY
}

\section{HOWARD WEINER}

\begin{abstract}
It is known that if $T$ is a one-to-one, measurable, invertible and nonsingular transformation on the unit interval with a $\sigma$-finite invariant measure, then its induced transformation $T_{1}$ on $L_{1}$ functions $f$ is such that $\lim _{n \rightarrow \infty} 1 / n \sum_{k=1}^{n} T_{1}^{k} f(x)$ exists. In this note, a counterexample is constructed which shows that the converse is false.
\end{abstract}

Ornstein [4] constructed a linear, piecewise affine transformation on the unit interval which has no $\sigma$-finite invariant measure. Chacon [1] accomplished the same objective by constructing a transformation $T$ whose induced transformation on $L_{1}$ functions $f$, denoted here by $T_{1}$, was such that

$$
\lim \inf _{n \rightarrow \infty} \frac{1}{n} \sum_{k=1}^{n} T_{1}^{k} f(x)=0 \text { a.e., and }
$$

$$
\lim \sup _{n \rightarrow \infty} \frac{1}{n} \sum_{k=1}^{n} T_{1}^{k} f(x)=\infty \text { a.e. , }
$$

since it is clear that $T$ cannot have a $\sigma$-finite invariant measure if the sequence $\left\{1 / n \sum_{k=1}^{n} T_{1}^{k} f(x)\right\}$ does not have a limit. (See also Jacobs [3].) The question arises as to whether the converse holds: if $\lim _{n \rightarrow \infty} 1 / n \sum_{k=1}^{n} T_{1}^{k} f(x)$ exists, then $T$ has a $\sigma$-finite invariant measure. It is the purpose of this paper to show that this statement is false by constructing a linear, piecewise affine transformation $T$ on the interval $I=(0,101 / 100]$ such that its induced transformation $T_{1}$ on $L_{1}$ functions $f$ satisfies

$$
\lim _{n \rightarrow \infty} \frac{1}{n} \sum_{k=1}^{n} T_{1}^{k} f(x)=0 \text { a.e. }
$$

Section 2 gives the construction of $T, \S 3$ contains the proof that $T$ has no $\sigma$-finite invariant measure, and $\S 4$ shows that the induced transformation, $T_{1}$, satisfies (2).

The author is indebted to D. Ornstein for suggesting the method of construction of $T$, which parallels his construction in [1]. (See also [3].)

2. Construction of $T$. The transformation of $T$ will be defined inductively step-by-step, and completely constructed in a denumerable number of steps. At each step, the domain of $T$ will be extended to a subinterval of $(1,101 / 100]$, and $T$ will not be altered where once 
defined.

At the first step, let $T$ take $(0,1 / 2]$ onto $(1 / 2,1]$ in an orderpreserving, affine way. Break up the interval $\left(1,1+(100)^{-1} / 2\right]$ into $10^{6}$ disjoint subintervals each of equal length $10^{-8} / 2$. Denote $(0,1 / 2]$ by $I_{1},(1 / 2,1]$ by $I_{2}$, and number the $10^{6}$ subintervals just defined left to right by $I_{3}, \cdots, I_{4}, \cdots, I_{10^{6}+2^{\circ}}$ Let $T$ take $I_{2}$ onto $I_{3}, I_{3}$ onto $I_{4}, \cdots, I_{16^{6}+1}$ onto $I_{16^{6}+2}$, in an order-preserving, affine way.

The domain of $T$ will now be extended to some part of $I_{16^{6}+2}$ using the method of [1]: split $I_{1}$ into two subintervals of equal length $I_{11}=(0,1 / 4]$ and $I_{12}=(1 / 4,1 / 2]:$ split $I_{2}=(1 / 2,1]$ into $I_{21}=(1 / 2,3 / 4]$ and $I_{22}=(3 / 4,1]$. Similarly define $I_{j 1}$ and $I_{j 2}$ for $3 \leqq j \leqq 10^{6}+2$. It is clear that $T$ already takes $I_{j 1}$ onto $I_{j+1,1}$ for $1 \leqq j \leqq 10^{6}+1$. Now split up all intervals $I_{j 2}, 1 \leqq j \leqq 10^{6}+2$ into $10^{3}$ subintervals of equal length. By an obvious left-to-right numbering scheme, $I_{j 2}$ will be the union of consecutive disjoint subintervals $I_{j, 2,1}, I_{j, 2,2}, \cdots, I_{j, 2,10^{3}}$ called the right part of $I_{j} . \quad I_{j 1}$ is called the left part of $I_{j}$. It is clear that $T$ already takes $I_{j, 2, l}$ onto $I_{j+1,2, l}$ for $1 \leqq j \leqq 10^{6}+1,1 \leqq l \leqq 10^{3}$ in an order-preserving, affine way.

The domain of $T$ will now be extended to the subinterval

$$
I_{16^{6}+2}-I_{16^{6}+2,2,10^{3}}=I_{10^{6}+2,1} \cup\left(\bigcup_{l=1}^{10^{3}-1} I_{16^{6}+2,2, l}\right) \text {, }
$$

as follows. Let $T$ take $I_{10^{6}+2,1}$ onto $I_{1,2,1}$ and $I_{10^{6}+2,2, l}$ onto $I_{1,2, l+1}$ for $1 \leqq l \leqq 10^{3}-1$ in an order-preserving, affine way. Now relabel all intervals from left to right $I_{1}, \cdots, I_{M M_{1}}$. This completes step one.

At the end of step $n-1$, relabelling the intervals in an obvious way, $T$ takes interval $I_{j}$ onto $I_{j+1}$ for $1 \leqq j \leqq M_{n}$ in an order-preserving, affine way. $T$ is not yet defined on $I_{M_{n}}$ and $T$ will now be defined on part of $I_{M_{n}}$. Split $I_{M_{n}}$ into $10^{6^{n}}$ subintervals of equal length, and order them from left to right as $I_{M_{n}}+1, \cdots, I_{N_{n}}$, where $N_{n}=M_{n}+10^{6^{n}}$. Now let $T$ take $I_{j}$ onto $I_{j+1}, M_{n} \leqq j \leqq N_{n}$ in an order-preserving, affine way. The domain of $T$ will now be extended to some part of $I_{N_{n}}$ using the method of [1].

For $1 \leqq j \leqq N_{n}$, split $I_{j}$ into two disjoint intervals of equal length, written $I_{j 1}$ and $I_{j 2}$, numbering from left to right. Divide the right interval $I_{j 2}$ into $10^{3^{n}}$ disjoint subintervals of equal length, and denote them, from left to right, by $I_{j, 2, l}, 1 \leqq j \leqq N_{n}$ and $1 \leqq l \leqq 10^{3^{n}}$. It is clear that $T$ already takes $I_{j, 1}$ onto $I_{j+1,1}$ and $I_{j, 2, l}$ onto $I_{j+1,2, l}$ for $1 \leqq j \leqq N_{n}-1$ and $1 \leqq l \leqq 10^{3^{n}}$. The domain of $T$ will now be extended to $I_{N_{n}}-I_{N_{n, 2,10^{3}}}=I_{N_{n}, 1} \bigcup\left(\bigcup_{l=1}^{10^{3 n}-1} I_{N_{n}, 2, l}\right)$. Let $T$ take $I_{N_{n}, 1}$ onto $I_{1,2,1}$ and $I_{N_{n}, 2, l}$ onto $I_{1,2, l+1}$ in an order-preserving affine way for $1 \leqq l \leqq 10^{3^{n}}-1$. This completes the definition of $T$ at the $n^{\text {th }}$ step. Now relabel all intervals from left to right as $I_{1}, I_{2}, \cdots, I_{M_{n+1}}$ to prepare for the $n+1^{\text {st }}$ step. 


\section{Invariance properties of $T$.}

Definition. ([1], [3]). Two sets, $E, F$, are said to be finitely $T$-equivalent if they allow finite disjoint decompositions $E=\sum_{k=1}^{n} E_{k}$ and $F=\sum_{k=1}^{n} F_{k}$, such that for appropriate $r_{k}, T^{r_{k}} E_{k}=F_{k}$.

THEOREM. T has no $\sigma$-finite invariant measure.

Proof. We let $m_{0}$ denote Lebesgue measure. It suffices to show that $T$ has the following property (See [1], [3] pp. 58-60, which this treatment follows):

For any integer $n$ and any set $M \subset I$, such that $m_{0}(M)>9 / 10$ there is a set of $n$ mutually disjoint and $T$-equivalent subsets $M_{1}, \cdots$, $M_{n}$ contained in $M$ such that $m_{0}\left(M_{1}\right)>1 / 8$.

To show that this property holds, it suffices to choose $M \subset(0,1]$ such that $m_{0}(M)>9 / 10$. At step $r$, suppose $U I \subset(0,1]$ where the union is taken only over those subintervals containing a subset of $M$. Renumber the subintervals $J_{1}, J_{2}, \cdots, J_{p}$, where $T$ or its positive powers takes $J_{l}$ onto $J_{l+1}, l=1,2, \cdots, P-1$. Suppose $E=\left\{l: J_{l} \subset \bigcup_{j=1}^{r-1} I_{j}\right\}$. By the construction, $m_{0}\left(\bigcup_{l \in E} J_{l}\right)=1 / 2$.

Let $L=\max \{l: l \in E\}$. Assume $r>n$. Then for $L<s \leqq P, J$ is in the right part of the scheme and hence

(3) $m_{0}\left(J_{s}\right) \leqq 10^{-6^{r}-3^{3 r}}<1 / 100 n L$, since $L \sim 10^{3 r}$. From this point on the proof is formally identical with that in [3], p. 60. This observation completes the proof.

\section{Convergence of Cesàro sums.}

Definition. The transformation on $L_{1}$ functions $f$ induced by $T$, denoted by $T_{1}$, is defined for $x_{0} \in(0,101 / 100]$ as

$$
T_{1} f(x)=f\left(T\left(x_{1}\right)\right) R\left(T, x_{0}, x_{1}\right),
$$

where $T\left(x_{1}\right)=x_{0}$ and $R\left(T, x_{0}, x_{1}\right)$ denotes the suitable Radon-Nikodym derivative of $T$ defined almost everywhere which insures that

$$
\int_{0}^{101 / 100} T_{1} f(x) d x=\int_{0}^{101 / 100} f(x) d x .
$$

$T_{1}$ is well defined. It is clear how to define powers of $T_{1}$. This may be expressed as $T_{1}^{n} f\left(x_{0}\right)=f\left(T^{n}\left(x_{1}\right)\right) R\left(T^{n}, x_{0}, x_{1}\right)$, where $T^{n}\left(x_{1}\right)=x_{0}$ and $R\left(T^{n}, x_{0}, x_{1}\right)$ denotes the Radon-Nikodym derivative which insures that

$$
\int_{0}^{101 / 100} T_{1}^{n} f(x) d x=\int_{0}^{101 / 100} f(x) d x
$$


Note that $R\left(T^{n}, x_{0}, x_{1}\right)$ is easy to compute. If $T^{n}\left(x_{0}\right)=x_{1}$ and $x_{0} \in I_{l}$ and $x_{1} \in I_{m}$, where $I_{l}$ and $I_{m}$ are intervals defined together in the same step in the definition of $T$ such that $m \neq l$, then

$$
R\left(T^{n}, x_{0}, x_{1}\right)=m_{0}\left(I_{l}\right) / m_{0}\left(I_{m}\right)=\text { length }\left(I_{l}\right) / \text { length }\left(I_{m}\right)
$$

due to the piecewise affine character of $T$.

In order to show that for $f \in L_{1}$,

$$
\lim _{n \rightarrow \infty} \frac{1}{n} \sum_{k=1}^{n} T_{1}^{k} f(x)=0 \text { a.e. } x \in I
$$

is suffices to show (5) only for $f=1$. This is so because if (5) holds for $f=1$, by the Chacon-Ornstein theorem [2], for any $g \in L_{1}$,

$$
\lim _{n \rightarrow \infty} \sum_{k=1}^{n} T_{1}^{k} g(x) / \sum_{k=1}^{n} T_{1}^{k} f(x) \text { exists a.e. } x \in I,
$$

and hence

$$
\lim _{n \rightarrow \infty} \frac{1}{n} \sum_{k=1}^{n} T_{1}^{k} g(x)=0 \text { a.e. } x \in I \text {. }
$$

Thus it suffices to prove the following.

THEOREM. For $f=1$

$$
\lim _{n \rightarrow \infty} \frac{1}{n} \sum_{k=1}^{n} T_{1}^{k} f(x)=0 \text { a.e. } x \in I .
$$

Proof. The proof is divided into two cases; (a) $x \in(0,1]$ and (b) $x \in(1,101 / 100]$.

Case (a). Recall that $M_{n}$ is the number of subintervals on which $T$ or its range was defined at step $n$. Note that the point $x=1$ is in the $R_{n}$-th interval at step $n$, where $R_{n}=M_{n}-\sum_{k=1}^{n} 10^{6 k}$.

Define $f_{n}(1)=1 / R_{n} \sum_{l=1}^{R_{n}} T_{1}^{l} f(1)$. Then $f_{n}(1)$ is clearly the Cesàro sum of highest index $\left(R_{n}\right)$ which can be defined at step $n$ at the point $x=1$ among the sums $1 / p \sum_{l=1}^{p} T_{1}^{l} f(1)$. Also, for $x \in(0,1], R_{n}$ is the maximum index $p$ such that $1 / p \sum_{l=1}^{p} T_{1}^{l} f(x)$ may be defined at step $n$.

Claim 1. $f_{n}(1) \leqq 10^{6^{n}} \times 0\left(10^{3^{n}}\right)$.

Proof. Proceeding by induction, we first obtain an upper bound for $f_{1}(1)$. The point $x=1$ is in interval $I_{M_{1}}-10^{6}$ which is of length $1 / 4 \times 10^{-3}$ and the intervals that map into $I_{1}$ at step 1 by $T$ or its positive powers are each of one of the following types:

Type 1. $I_{1}, I_{2}$ each of length $1 / 4$, and hence each contributing $10^{3}$. 
to the sum $\sum_{l=1}^{R_{n}} T_{1}^{l} f(1)$ by (4);

Type 2. $I_{3}, \cdots, I_{10^{6}+2}$, each of length $1 / 4 \times 10^{-8}$, and so by (4) each contributing $10^{-5}$ to the above sum;

Type 3. $I_{10^{6}+3}, I_{10^{6}+4}, I_{2 \times 10^{6}+5}, I_{2 \times 10^{6}+6}, I_{3 \times 10^{6}+7}, I_{3^{\times} 1_{10}+8}, \cdots, I_{\left(10^{3}-1\right) 10^{6}+2\left(10^{3}-1\right)+1}$, $I_{\left(10^{3}-1\right)^{1} 0^{6}+2\left(10^{3}-1^{\prime}+2\right.}$, each of length $1 / 4 \times 10^{-3}$, and hence each contributing 1 to the sum; and

Type 4. $I_{10_{6+5}}, \cdots, I_{2 \times 10^{6}+4}, I_{2 \times 10^{6}+7}, I_{3^{\times} 0^{6}+6}, \cdots, I_{\left(10^{3}-1\right) \times 10^{6}+2\left(10^{3}-1\right)+3}, \cdots$, $I_{10^{3} \times\left(10^{6}\right)+2\left(10^{3}-1\right)+3}$, each of length $1 / 4 \times 10^{-11}$, and hence contributing $10^{-8}$ to the sum.

Multiplying the contribution of each type of interval by a number at least as large as the number of each such interval, adding these four terms, and dividing by a number smaller than the total number of summands $R_{n}$ yields the following upper bound

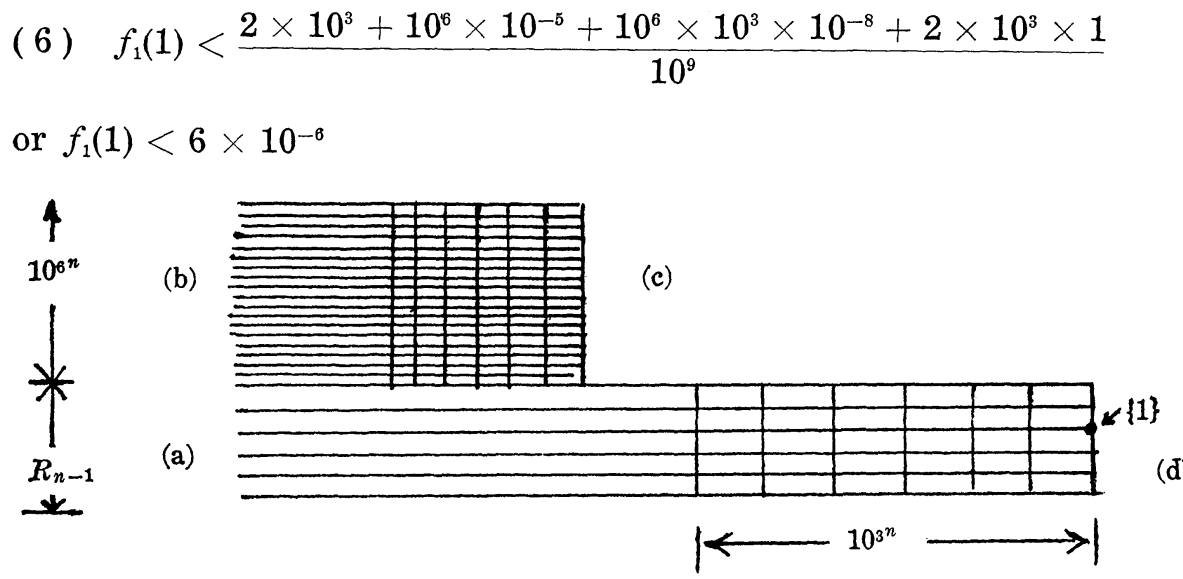

FIgURE 1.

Consider the above diagram representing the four types of domain of definition on which $T$ and its positive powers are defined at step $n$. The domain (a) is the set of left parts of $(0,1]$ together with the left parts of the subintervals of $(1,101 / 100]$ added to the domain before step $n$. Domain (b) is the set of left parts of the subinterval of (1, 101/100] added to the domain of definition of $T$ at step $n$. Domain (c) is the right part of the subinterval added to the domain of $T$ at step $n$. Domain (d) is the right part of $(0,1]$ together with the right part of the subintervals of $(1,101 / 100]$ added to the domain before step $n$. The numbers on the diagram refer to the respective number of subintervals into which the left parts right parts, of $(0,1]$ and appropriate subintervals of $(1,101 / 100]$ are divided at the $n^{\text {th }}$ step.

Using an obvious notation,

$$
R_{n} f_{n}(1)=\sum_{l=1}^{R_{n}} T_{1}^{l} f(1)=\sum_{(\mathrm{a})}+\sum_{(\mathrm{b})}+\sum_{(\mathrm{c})}+\sum_{(\mathrm{d})} T_{1}^{l} f(1),
$$


where

$$
\sum_{(a)} T_{1}^{l} f(1)=f_{n-1}(1) R_{n-1} \times 10^{3^{n}},
$$

since the length ratio of left part intervals to the corresponding right part intervals is $10^{3 n}$;

$$
\begin{aligned}
\sum_{\text {(b) }} T_{1}^{l} f(1) & =10^{-6^{n}} \times 2^{-n} \times \frac{1}{200} \times 2^{n} \times 10^{\Sigma_{j=1}^{n} 3^{j}} \times 10^{6^{n}} \\
& =\frac{1}{200} \times 10^{\Sigma_{j=1^{3}}^{n}},
\end{aligned}
$$

where $10^{-6^{n}} \times 2^{-n} \times 1 / 200$ is the length of a (b) interval, $2^{-n} \times 10^{-\Sigma_{j=1^{n}}^{n}}$ is the length of the (d) interval containing the point 1 , and $10^{6^{n}}$ is the number of (b) intervals;

$$
\begin{aligned}
\sum_{\text {(c) }} T_{1}^{l} f(1)= & \frac{1}{200} \times 2^{-n} \times 10^{-6^{n}} \times 10^{-3^{n}} \times\left[10^{\Sigma_{j=1^{3^{j}}}^{n}} \times 2^{n}\right] \\
& \times 10^{6^{n}} \times 10^{3^{n}}=\frac{1}{200} \times 10^{\Sigma_{j=1^{3}}^{n}},
\end{aligned}
$$

since each subinterval of (c) has length $\left((100) \times 2^{n+1} \times 10^{6 n+3 n}\right)^{-1}$, the subinterval containing the point 1 has length $\left(10^{\Sigma_{j=1^{3}}^{n}} \times 2^{n}\right)^{-1}$ and there are a total of $10^{6^{n}+3^{n}}$ subintervals in (c);

$$
\sum_{(d)} T_{1}^{l} f(1)<R_{n-1} f_{n-1}(1) \times 10^{3^{n}}
$$

since there are $10^{3 n}$ sets of intervals on which $T$ and its positive powers were defined at the $n-1^{\text {st }}$ step in (d).

Clearly

$$
R_{n}<10^{6^{n}+3^{n}}
$$

Hence from (6) - (12) inclusive,

$$
f_{n}(1)<\frac{2 f_{n-1}(1) \times R_{n-1} \times 10^{3^{n}}+2 \times\left(\frac{1}{200}\right) \times 10^{\Sigma_{j=1^{3}}^{n}}}{10^{6^{n}+2^{n}}} .
$$

By the induction hypothesis, $f_{n-1}(1)=10^{-6^{n-1}} \times 0\left(10^{3 n-1}\right)$.

Using this in (13), $f_{n}(1)<10^{-6^{n}} \times 0\left(10^{3^{n}}\right)$, completing the induction argument.

Now consider $x \in(0,1]$ such that in addition, $x$ is in the right part of the scheme. In the diagram below, at step $n$, the second subinterval in the right part of the scheme which is also a subinterval of $(0,1]$ is denoted by $Q$. This interval is $I_{r_{0}}$, where $r_{0}=$ $M_{n-1}+10^{6^{n}}+1>10^{6^{n}}$. Let $x_{0} \in Q$. 
(b)

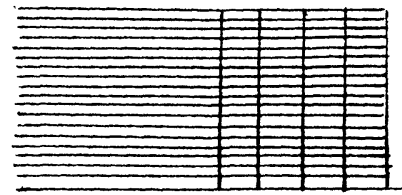

(d)

(a)

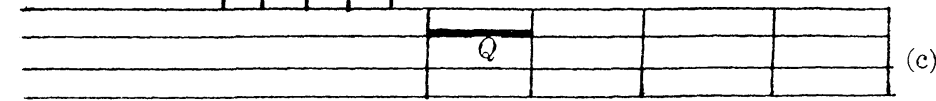

FIGURE 2.

Claim. Suppose $r$ is such that $M_{n}>r>M_{n-1}$, and $x \in(0,1]$ and also in the right part of the scheme at step $n$. Then under these conditions,

$$
\max _{x, r} \frac{1}{r} \sum_{l=1}^{r} T_{1}^{l} f(x)=\frac{1}{r_{0}} \sum_{l=1}^{r_{n}} f\left(x_{0}\right) .
$$

This is clear since the largest Radon-Nikodym derivatives in the above Cesàro sum come about as a result of $T$ and its positive powers taking points from the left part of the scheme to its right part.

Claim 2. At step $n$,

$$
\frac{1}{r_{0}} \sum_{l=1}^{r_{0}} T_{1}^{l} f\left(x_{0}\right)<f_{n-1}(1) .
$$

Proof. From the above diagram,

$$
\sum_{l=1}^{r_{0}} T_{1}^{l} f\left(x_{0}\right)=\sum_{(\mathrm{a})}+\sum_{(\mathrm{b})}+\sum_{(\mathrm{c})} T_{1}^{l} f\left(x_{\mathrm{c}}\right),
$$

where

$$
\sum_{\text {(a) }} T_{1}^{l} f\left(x_{0}\right)=10^{3 n} \times M_{n-1} \times f_{n-1}(1)
$$

$$
\sum_{(\mathrm{b})} T_{1}^{l} f\left(x_{0}\right)=(200)^{-1} \times 2^{-n} \times 10^{-6^{n}} \times\left(10^{\Sigma_{j=1^{3^{j}}}} \times 2^{n}\right) \times 10^{6^{n}}<10^{n 3^{n}},
$$

and,

$$
\sum_{(c)} T_{1}^{l} f\left(x_{0}\right)=M_{n-1} \times f_{n-1}(1) .
$$

Hence from (15) - (19),

$$
\begin{aligned}
\frac{1}{r_{0}} \sum_{l=1}^{r_{0}} T_{1}^{l} f\left(x_{0}\right) & <\frac{\left(10^{3^{n}}+1\right) \times M_{n-1} \times f_{n-1}(1)+10^{n 3^{n}}}{10^{6^{n}}} \\
& \sim \frac{\left(10^{3^{n}}\right) \times 10^{3^{n-1}+6^{n-1}} \times f_{n-1}(1)+10^{n 3^{n}}}{10^{6^{n}}}<f_{n-1}(1) .
\end{aligned}
$$

This establishes the claim. 
Now a.e. $x \in(0,1]$ is in the right part of the scheme for infinitely many steps $n$ since at each step, every subinterval is divided into two equal subintervals, one of which becomes a member of the left part of the scheme, and the other, the right part. Further, higher powers of $T_{1}^{l} f(x)$ can only be defined at a given stage $n$ if $x$ is in the right part of the scheme. These remarks plus Claims 1 and 2 above establish that

$$
\lim _{r \rightarrow \infty} \frac{1}{r} \sum_{l=1}^{r} T_{1}^{l} f(1) \longrightarrow 0 \text { for a.e. } x \in(0,1]
$$

which is case (a).

For case $(b)$, let $x \in(1,101 / 100]$. The procedure to be followed parallels that in case (a).

$$
\text { Define } f_{1 k}\left(1+(100)^{-1} \times \sum_{l=1}^{r} 2^{-j}\right)=\frac{1}{M_{r_{k}}} \sum_{l=1}^{M_{r k}} T_{1}^{l} f\left(1+(100)^{-1} \times \sum_{j=1}^{r} 2^{-j}\right) \text {, }
$$

where $k \geqq r+1$ and $M_{r_{k}}=M_{k}-\sum_{j=r+1}^{k} 10^{6^{j}}$. That is, $M_{r_{k}}$ is the highest power of $T$ that may be defined at step $k$ with domain on a part of the $r^{\text {th }}$ subinterval $\left(1+(100)^{-1} \times 2^{-r}, 1+(100)^{-1} \times 2^{-r+1}\right]$ which is taken from $(1,101 / 100]$.

$$
\text { Claim 3. } f_{1 k}\left(1+(100)^{-1} \times \sum_{j=1}^{r} 2^{-j}\right)=10^{-6^{k}} \times 0\left(10^{3^{k-1}}\right)^{\rightarrow 0} \rightarrow 0
$$

for fixed $r$ as $k \rightarrow \infty$.

Claim 4. Let

$$
\left.x \in\left(1+(100)^{-1} \times \sum_{j=1}^{r-1} 2^{-j}\right), 1+(100)^{-1} \times \sum_{j=1}^{r} 2^{-j}\right] .
$$

Suppose that at step $k>r, x$ is in the right part of the scheme. Then for

$$
M>N_{k-1}, \frac{1}{M} \sum_{l=1}^{M} T_{1}^{l} f(x)<f_{1, k-1}\left(1+(00)^{-1} \times \sum_{j=1}^{r} 2^{-j}\right) .
$$

The proof of Claim 3 follows as for Claim 1, and that for Claim 4 as for Claim 2. The proofs use the fact that $10^{6 n} \gg 10^{3 n}$ as $n$ increases. The details are omitted.

Since a.e. $x \in(1,101 / 100]$ is in the right part of the scheme for infinitely many steps $n$, and since higher powers of $T_{1}^{l} f(x)$, for fixed $x$, are defined when $x$ is in the right part of the scheme at some step, Claims 3 and 4 yield the result for case (b). 


\section{REFERENCES}

1. R. V. Chacon, A class of linear transformations, Proc. Amer. Math. Soc. 15 (1964), 560-564.

2. R. V. Chacon, and D.S. Ornstein, A general ergodic theorem, Illinois J. Math. (2) 4 (1960), 153-160.

3. K. Jacobs, Lectures on ergodic theory, Matematisk Institut, Aarhus University Vol. 1 (1963), 56-60.

4. D. S. Ornstein, On invariant measures, Bull. Amer. Math. Soc. (4) 66 (1960), 297300 .

Received July 6, 1967.

UNiversity of California at Davis 



\title{
PACIFIC JOURNAL OF MATHEMATICS
}

\author{
EDITORS
}

\section{H. ROYDEN}

Stanford University

Stanford, California

\author{
J. P. JANS \\ University of Washington \\ Seattle, Washington 98105
}

\section{J. DugundJI}

Department of Mathematics

University of Southern California

Los Angeles, California 90007

\section{RICHARD ARENS}

University of California

Los Angeles, California 90024

\section{ASSOCIATE EDITORS}
E. F. BECKENBACH
B. H. NEUMANN
F. WOLF
K. YOSIDA

\section{SUPPORTING INSTITUTIONS}

\author{
UNIVERSITY OF BRITISH COLUMBIA \\ CALIFORNIA INSTITUTE OF TECHNOLOGY \\ UNIVERSITY OF CALIFORNIA \\ MONTANA STATE UNIVERSITY \\ UNIVERSITY OF NEVADA \\ NEW MEXICO STATE UNIVERSITY \\ OREGON STATE UNIVERSITY \\ UNIVERSITY OF OREGON \\ OSAKA UNIVERSITY \\ UNIVERSITY OF SOUTHERN CALIFORNIA
}

\author{
STANFORD UNIVERSITY \\ UNIVERSITY OF TOKYO \\ UNIVERSITY OF UTAH \\ WASHINGTON STATE UNIVERSITY \\ UNIVERSITY OF WASHINGTON \\ AMERICAN MATHEMATICAL SOCIETY \\ CHEVRON RESEARCH CORPORATION \\ TRW SYSTEMS \\ NAVAL WEAPONS CENTER
}

Mathematical papers intended for publication in the Pacific Journal of Mathematics should be in typed form or offset-reproduced, double spaced with large margins. Underline Greek letters in red, German in green, and script in blue. The first paragraph or two must be capable of being used separately as a synopsis of the entire paper. It should not contain references to the bibliography. Manuscripts, in duplicate if possible, may be sent to any one of the four editors. All other communications to the editors should be addressed to the managing editor, Richard Arens, University of California, Los Angeles, California 90024.

Each author of each article receives 50 reprints free of charge; additional copies may be obtained at cost in multiples of 50 .

The Pacific Journal of Mathematics is published monthly. Effective with Volume 16 the price per volume (3 numbers) is $\$ 8.00$; single issues, $\$ 3.00$. Special price for current issues to individual faculty members of supporting institutions and to individual members of the American Mathematical Society: $\$ 4.00$ per volume; single issues $\$ 1.50$. Back numbers are available.

Subscriptions, orders for back numbers, and changes of address should be sent to Pacific Journal of Mathematics, 103 Highland Boulevard, Berkeley 8, California.

Printed at Kokusai Bunken Insatsusha (International Academic Printing Co., Ltd.), 7-17, Fujimi 2-chome, Chiyoda-ku, Tokyo, Japan.

PUBLISHED BY PACIFIC JOURNAL OF MATHEMATICS, A NON-PROFIT CORPORATION

The Supporting Institutions listed above contribute to the cost of publication of this Journal, but they are not owners of publishers and have no responsibility for its content or policies. 


\section{Pacific Journal of Mathematics}

\section{Vol. 25, No. $3 \quad$ November, 1968}

Philip Marshall Anselone and Theodore Windle Palmer, Collectively

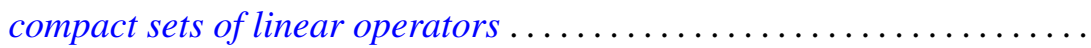

Philip Marshall Anselone and Theodore Windle Palmer, Spectral analysis of collectively compact, strongly convergent operator sequences.........

Edward A. Bender, Characteristic polynomials of symmetric matrices...... 433

Robert Morgan Brooks, The structure space of a commutative locally convex

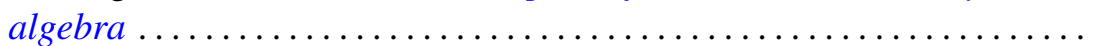

Jacob Feldman and Frederick Paul Greenleaf, Existence of Borel

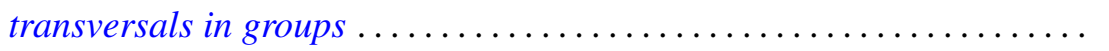

Thomas Muirhead Flett, Mean values of power series 463

Richard Vernon Fuller, Relations among continuous and various

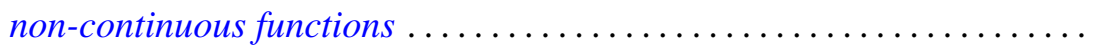

Philip Hartman, Convex sets and the bounded slope condition ............

Marcel Herzog, On finite groups containing a CCT-subgroup with a cyclic Sylow subgroup .

James Secord Howland, On the essential spectrum of Schroedinger

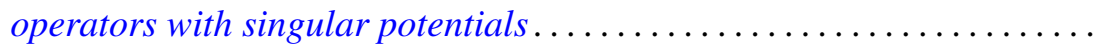

Thomas William Hungerford, On the structure of principal ideal rings .....

Paul Joseph Kelly and Ernst Gabor Straus, Curvature in Hilbert geometries. II mpati Madhusudana Rao, Linear functionals on Orlicz spaces:

Malempati Madhusudana Rao, Linear functionals on Orli
$\quad$ General theory............................
Stanley F. Robinson, Theorems on Brewer sums.......
Ralph Tyrrell Rockafellar, A general correspondence betw

Malempati Madhusudana Rao, Linear functiona
$\quad$ General theory.........................
Stanley F. Robinson, Theorems on Brewer sums
Ralph Tyrrell Rockafellar, A general corresponden

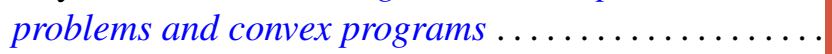

Richard Benjamin Sher, Defining subsets of $E^{3}$ by cubes.

Howard Jacob Weiner, Invariant measures and Cesàro summability... 